International Journal of Modern Physics E,

(C) World Scientific Publishing Company

\title{
A QUANTUM KINETIC EQUATION FOR PARTICLE PRODUCTION IN THE SCHWINGER MECHANISM
}

\author{
S. SCHMIDT*, D. BLASCHKE and G. RÖPKE \\ Fachbereich Physik, Universität Rostock, D- 18051 Rostock, Germany \\ S.A. SMOLYANSKY and A.V. PROZORKEVICH \\ Physics Department, Saratov State University, Saratov, Russia \\ V.D. TONEEV円 \\ Bogoliubov Laboratory of Theoretical Physics, Joint Institute \\ for Nuclear Research, 141980 Dubna, Russia
}

\begin{abstract}
A quantum kinetic equation is derived for the description of pair production in a timedependent homogeneous electric field $E(t)$. As a source term, the Schwinger mechanism for particle creation is incorporated. Possible particle production due to collisions and collisional damping are neglected. The main result is a kinetic equation of non-Markovian character. In the low density approximation, the source term is reduced to the leading part of the well known Schwinger formula for the probability of pair creation. We discuss the momentum and time dependence of the derived source term and compare with other approaches.
\end{abstract}

\section{Introduction}

Particle production in ultrarelativistic heavy-ion collisions raises a number of challenging problems. One of these interestipe creation into a kinetic theory $\mathrm{A}$, a lot of promising research has been carried out during the last years. In the scenario where a chromo-electric field is generated by a nucleus mage collision, the production of parton pairs can be described by the Schwinger mechanism 13, 14, 15. The produced charged particles generate a field which, in turn, modifies the initial electric field and may cause plasma oscillations. The interesting questim the back reaction of this field has been analyzed within a field

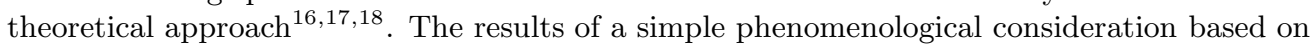
kinetic equations and the field-theoretical treatment 5.19 , 20.21 agree with each other. The source term which occurs in such a modified Boltzmann equation was derived phenomenologically in Ref.22. However, the systematic derivation of this source term in relativistic transport theory

*email: basti@darss.mpg.uni-rostock.de

${ }^{\dagger}$ Present address: Gesellschaft für Schwerionenforschung, Darmstadt, Germany 
is not yet fully carried out. Forpxample, recently it was pointed out that the source term may have non-Markovian character 6.23 even for the case of a constant electric field.

In the present work, a kinetic equation is derived in a consistent field theoretical treatment for the time evolution of the pair creation in a time-dependent and spatially homogeneous electric field. This derivation is based on the Bogoliubov transformation for field operators between the asymptotic in-state and the ifftantaneous state. In contrast with phenomenological approaches, but in agreement with 63 , the source term of the particle production is of non-Markovian character. The kinetic equation derived reproduces the Schwinger result in the low density approximation in the weak field limit.

The paper is organized as follows. In Section 2 we derive the kinetic equation with a new source term that describes particle production in a strong electric field within a field theoretical approach for both fermions and bosons. In Section 3 we discuss the main properties of the result and give numerical estimates for the case of a constant field and a model time dependent field. We summarize our results in Section 4.

\section{Dynamics of pair creation}

\subsection{Creation of fermion pairs}

In this section we demonstrate the derivation of a kinetic equation with a source term for fermion-antifermion production. As an illustrative example we consider electron-positron creation, however the generalization to quark-antiquark pair creation in a chromoelectric field in Abelian approximation is straightforward. For the description of $e^{+} e^{-}$production in an electric field we start from the QED lagrangian

$$
\mathcal{L}=\bar{\psi} i \gamma^{\mu}\left(\partial_{\mu}+i e A_{\mu}\right) \psi-m \bar{\psi} \psi-\frac{1}{4} F_{\mu \nu} F^{\mu \nu},
$$

where $F^{\mu \nu}$ is the field strength, the metric istaken as $g^{\mu \nu}=\operatorname{diag}(1,-1,-1,-1)$ and for the $\gamma-$ matrices we use the conventional definition 24. In the following we consider the electromagnetic field as classical and quantize only the matter field. Then the Dirac equation reads

$$
\left(i \gamma^{\mu} \partial_{\mu}-e \gamma^{\mu} A_{\mu}-m\right) \psi(x)=0 .
$$

We use a simple field-theoretical model to treat charged fermions in an external electric field charactarized by the vector potential $A_{\mu}=(0,0,0, A(t))$ with $A(t)=A_{3}(t)$. The electric field

$$
E(t)=E_{3}(t)=-\dot{A}(t)=-d A(t) / d t
$$

is assumed to be time-dependent but homogeneous in space $\left(E_{1}=E_{2}=0\right)$. This quasiclassical electric field interacts with a spinor field $\psi$ of fermions. We look for solutions of the Dirac equation where eigenstates are represented in the form

$$
\psi_{\bar{p} r}^{( \pm)}(x)=\left[i \gamma^{0} \partial_{0}+\gamma^{k} p_{k}-e \gamma^{3} A(t)+m\right] \chi^{( \pm)}(\bar{p}, t) R_{r} \mathrm{e}^{i \bar{p} \bar{x}}
$$

where $k=1,2,3$ and the superscript $( \pm)$ denotes eigenstates with the positive and negative frequencies. Herein $R_{r}(r=1,2)$ is an eigenvector of the matrix $\gamma^{0} \gamma^{3}$

$$
R_{1}=\left(\begin{array}{r}
0 \\
1 \\
0 \\
-1
\end{array}\right), \quad R_{2}=\left(\begin{array}{r}
1 \\
0 \\
-1 \\
0
\end{array}\right)
$$


so that $R_{r}^{+} R_{s}=2 \delta_{r s}$. The functions $\chi^{( \pm)}(\bar{p}, t)$ are related to the oscillator-type equation

$$
\ddot{\chi}^{( \pm)}(\bar{p}, t)=-\left(\omega^{2}(\bar{p}, t)+i e \dot{A}(t)\right) \chi^{( \pm)}(\bar{p}, t),
$$

where we define the total energy $\omega^{2}(\bar{p}, t)=\varepsilon_{\perp}^{2}+P_{\|}^{2}(t)$, the transverse energy $\varepsilon_{\perp}^{2}=m^{2}+\bar{p}_{\perp}^{2}$ and $P_{\|}(t)=p_{\|}-e A(t)$. The solutions $\chi^{( \pm)}(\bar{p}, t)$ of Eq. (1) for positive and negative frequencies are defined by their asymptotic behavior at $t_{0}=t \rightarrow-\infty$, where $A\left(t_{0}\right)=0$. We obtain

$$
\chi^{( \pm)}(\bar{p}, t) \sim \exp \left( \pm i \omega_{0}(\bar{p}) t\right)
$$

where the total energy in asymptotic limit is given as $\omega_{0}(\bar{p})=\omega_{0}\left(\bar{p}, t_{0}\right)=\lim _{t \rightarrow-\infty} \omega(\bar{p}, t)$. Note that the system of the spinor functions (4) is complete and orthonormalized. The field operators $\psi(x)$ and $\bar{\psi}(x)$ can be decomposed in the spinor functions (ब) as follows:

$$
\psi(x)=\sum_{r, \bar{p}}\left[\psi_{\bar{p} r}^{(-)}(x) b_{\bar{p} r}\left(t_{0}\right)+\psi_{\bar{p} r}^{(+)}(x) d_{-\bar{p} r}^{+}\left(t_{0}\right)\right] .
$$

The operators $b_{\bar{p} r}\left(t_{0}\right), b_{\bar{p} r}^{+}\left(t_{0}\right)$ and $d_{\bar{p} r}\left(t_{0}\right), d_{\bar{p} r}^{+}\left(t_{0}\right)$ describe the creation and annihilation of electrons and positrons in the in-state $\left|0_{i n}\right\rangle$ at $t=t_{0}$, and satisfy the anti-commutation relations 24

$$
\left\{b_{\bar{p} r}\left(t_{0}\right), b_{\bar{p}^{\prime} r^{\prime}}^{+}\left(t_{0}\right)\right\}=\left\{d_{\bar{p} r}\left(t_{0}\right), d_{\bar{p}^{\prime} r^{\prime}}^{+}\left(t_{0}\right)\right\}=\delta_{r r^{\prime}} \delta_{\bar{p} \bar{p}^{\prime}} .
$$

The evolution affects the vacuum state and mixes states with positive and negative energies resulting in non-diagonal terms that are responsible for pair creation. The diagonalization of the hamiltonian corresponding to a Dirac-particle (Eq. (2)) in the homogeneous electric field (3) is achieved by a time-dependent Bogoliubov transformation

$$
\begin{aligned}
& b_{\bar{p} r}(t)=\alpha_{\bar{p}}(t) b_{\bar{p} r}\left(t_{0}\right)+\beta_{\bar{p}}(t) d_{-\bar{p} r}^{+}\left(t_{0}\right), \\
& d_{\bar{p} r}(t)=\alpha_{-\bar{p}}(t) d_{\bar{p} r}\left(t_{0}\right)-\beta_{-\bar{p}}(t) b_{-\bar{p} r}^{+}\left(t_{0}\right)
\end{aligned}
$$

with the condition

$$
\left|\alpha_{\bar{p}}(t)\right|^{2}+\left|\beta_{\bar{p}}(t)\right|^{2}=1 \text {. }
$$

Here, the operators $b_{\bar{p} r}(t)$ and $d_{\bar{p} r}(t)$ describe the creation and annihilation of quasiparticles at the time $t$ with the instantaneous vacuum $\left|0_{t}\right\rangle$. Clearly, the operator system $b\left(t_{0}\right), b^{+}\left(t_{0}\right) ; d\left(t_{0}\right), d^{+}\left(t_{0}\right)$ is unitary non-equivalent to the system $b(t), b^{+}(t) ; d(t), d^{+}(t)$. The substitution of Eqs. (10) into Eq. (8) leads to the new representation of the field operators

$$
\psi(x)=\sum_{r, \bar{p}}\left[\Psi_{\bar{p} r}^{(-)}(x) b_{\bar{p} r}(t)+\Psi_{\bar{p} r}^{(+)}(x) d_{-\bar{p} r}^{+}(t)\right] .
$$

The link between the new $\Psi_{\bar{p} r}^{( \pm)}(x)$ and the former (ब) basis functions is given by a canonical transformation

$$
\begin{aligned}
\psi_{\bar{p} r}^{(-)}(x) & =\alpha_{\bar{p}}(t) \Psi_{\bar{p} r}^{(-)}(x)-\beta_{\bar{p}}^{*}(t) \Psi_{\bar{p} r}^{(+)}(x), \\
\psi_{\bar{p} r}^{(+)}(x) & =\alpha_{\bar{p}}^{*}(t) \Psi^{(+)}(x)_{\bar{p} r}+\beta_{\bar{p}}(t) \Psi_{\bar{p} r}^{(-)}(x) .
\end{aligned}
$$


Therefore it is justified to assume that the functions $\Psi_{\bar{p} r}^{( \pm)}$have a spin structure similar to that of $\psi_{\bar{p} r}^{(+)}$in Eq. (目),

$$
\Psi_{\bar{p} r}^{( \pm)}(x)=\left[i \gamma^{0} \partial_{0}+\gamma^{k} p_{k}-e \gamma^{3} A(t)+m\right] \phi_{\bar{p}}^{( \pm)}(x) R_{r} \mathrm{e}^{ \pm i \Theta(t)} \mathrm{e}^{i \bar{p} \bar{x}}
$$

where the dynamical phase is defined as

$$
\Theta(\bar{p}, t)=\int_{t_{0}}^{t} d t^{\prime} \omega\left(\bar{p}, t^{\prime}\right)
$$

The functions $\phi_{\bar{p}}^{( \pm)}$are yet unknown. The substitution of Eq. (14) into Eqs. (13) leads to the relations

$$
\begin{aligned}
\chi^{(-)}(\bar{p}, t) & =\alpha_{\bar{p}}(t) \phi_{\bar{p}}^{(-)}(t) \mathrm{e}^{-i \Theta(\bar{p}, t)}-\beta_{\bar{p}}^{*}(t) \phi_{\bar{p}}^{(+)}(t) \mathrm{e}^{i \Theta(\bar{p}, t)}, \\
\chi^{(+)}(\bar{p}, t) & =\alpha_{\bar{p}}^{*}(t) \phi_{\bar{p}}^{(+)}(t) \mathrm{e}^{i \Theta(\bar{p}, t)}+\beta_{\bar{p}}(t) \phi_{\bar{p}}^{(-)}(t) \mathrm{e}^{-i \Theta(\bar{p}, t)} .
\end{aligned}
$$

Now we are able to find explicit expressions for the coefficients $\alpha_{\bar{p}}(t)$ and $\beta_{\bar{p}}(t)$. Taking into account that the functions $\chi^{( \pm)}(\bar{p}, t)$ are defined by Eq. (6), we introduce additional conditions to Eqs. (16) according to the Lagrange method

$$
\begin{aligned}
& \dot{\chi}^{(-)}(\bar{p}, t)=-i \omega(\bar{p}, t)\left[\alpha_{\bar{p}}(t) \phi_{\bar{p}}^{(-)}(t) \mathrm{e}^{-i \Theta(\bar{p}, t)}+\beta_{\bar{p}}^{*}(t) \phi_{\bar{p}}^{(+)}(t) \mathrm{e}^{i \Theta(\bar{p}, t)}\right], \\
& \dot{\chi}^{(+)}(\bar{p}, t)=i \omega(\bar{p}, t)\left[\alpha_{\bar{p}}^{*}(t) \phi_{\bar{p}}^{(+)}(t) \mathrm{e}^{i \Theta(\bar{p}, t)}-\beta_{\bar{p}}(t) \phi_{\bar{p}}^{(-)}(t) \mathrm{e}^{-i \Theta(\bar{p}, t)}\right] .
\end{aligned}
$$

Differentiating these equations with respect to time, using Eqs. (6) and (16) and then choosing as an Ansatz

$$
\phi_{\bar{p}}^{( \pm)}(t)=\sqrt{\frac{\omega(\bar{p}, t) \pm P_{\|}(t)}{\omega(\bar{p}, t)}},
$$

we obtain the following differential equations for the coefficients

$$
\begin{aligned}
& \dot{\alpha}_{\bar{p}}(t)=\frac{e E(t) \varepsilon_{\perp}}{2 \omega^{2}(\bar{p}, t)} \beta_{\bar{p}}^{*}(t) \mathrm{e}^{2 i \Theta(\bar{p}, t)}, \\
& \dot{\beta}_{\bar{p}}^{*}(t)=-\frac{e E(t) \varepsilon_{\perp}}{2 \omega^{2}(\bar{p}, t)} \alpha_{\bar{p}}(t) \mathrm{e}^{-2 i \Theta(\bar{p}, t)} .
\end{aligned}
$$

As the result of the Bogoliubov transformation we obtained the new coefficients of the instantaneous state at the time $t$. The relations between them read

$$
\begin{aligned}
& \alpha_{\bar{p}}(t)=\frac{1}{2 \sqrt{\omega(\bar{p}, t)\left(\omega(\bar{p}, t)-P_{\|}(t)\right)}}\left(\omega(\bar{p}, t) \chi^{(-)}(\bar{p}, t)+i \dot{\chi}^{(-)}(\bar{p}, t)\right) \mathrm{e}^{i \Theta(\bar{p}, t)}, \\
& \beta_{\bar{p}}^{*}(t)=-\frac{1}{2 \sqrt{\omega(\bar{p}, t)\left(\omega(\bar{p}, t)-P_{\|}(t)\right)}}\left(\omega(\bar{p}, t) \chi^{(-)}(\bar{p}, t)-i \dot{\chi}^{(-)}(\bar{p}, t)\right) \mathrm{e}^{-i \Theta(\bar{p}, t)} .
\end{aligned}
$$


It is convenient to introduce new operators which absorb the dynamical phase

$$
\begin{aligned}
& B_{\bar{p} r}(t)=b_{\bar{p} r}(t) e^{-i \Theta(\bar{p}, t)}, \\
& D_{\bar{p} r}(t)=d_{\bar{p} r}(t) e^{-i \Theta(\bar{p}, t)}
\end{aligned}
$$

satisfying the anti-commutation relations:

$$
\left\{B_{\bar{p} r}(t), B_{\bar{p}^{\prime} r^{\prime}}^{+}(t)\right\}=\left\{D_{\bar{p} r}(t), D_{\bar{p}^{\prime} r^{\prime}}^{+}(t)\right\}=\delta_{r r^{\prime}} \delta_{\bar{p} \bar{p}^{\prime}} .
$$

It is easy to show that these operators satisfy the Heisenberg-like equations of motion

$$
\begin{aligned}
& \frac{d B_{\bar{p} r}(t)}{d t}=-\frac{e E(t) \varepsilon_{\perp}}{2 \omega^{2}(\bar{p}, t)} D_{-\bar{p} r}^{+}(t)+i\left[H(t), B_{\bar{p} r}(t)\right], \\
& \frac{d D_{\bar{p} r}(t)}{d t}=\frac{e E(t) \varepsilon_{\perp}}{2 \omega^{2}(\bar{p}, t)} B_{-\bar{p} r}^{+}(t)+i\left[H(t), D_{\bar{p} r}(t)\right],
\end{aligned}
$$

where $H(t)$ is the hamiltonian of the quasiparticle system

$$
H(t)=\sum_{r, \bar{p}} \omega(\bar{p}, t)\left(B_{\bar{p} r}^{+}(t) B_{\bar{p} r}(t)-D_{-\bar{p} r}(t) D_{-\bar{p} r}^{+}(t)\right) .
$$

The first term on the r.h.s. of Eqs. (24) is caused by the unitary non-equivalence of the in-representation and the quasiparticle one.

Now we explore the evolution of the distribution function of electrons with the momentum $\bar{p}$ and spin $r$ defined as

$$
f_{r}(\bar{p}, t)=<0_{i n}\left|b_{\bar{p} r}^{+}(t) b_{\bar{p} r}(t)\right| 0_{i n}>=<0_{i n}\left|B_{\bar{p} r}^{+}(t) B_{\bar{p} r}(t)\right| 0_{i n}>.
$$

According to the charge conservation the distribution functions for electrons and positrons are equal $f_{r}(\bar{p}, t)=\bar{f}_{r}(\bar{p}, t)$, where

$$
\bar{f}_{r}(\bar{p}, t)=<0_{i n}\left|d_{-\bar{p} r}^{+}(t) d_{-\bar{p} r}(t)\right| 0_{i n}>=<0_{i n}\left|D_{-\bar{p} r}^{+}(t) D_{-\bar{p} r}(t)\right| 0_{i n}>.
$$

The distribution functions (26) and (27) are normalized to the total number of pairs $N(t)$ of the system at a given time $t$

$$
\sum_{r, \bar{p}} f_{r}(\bar{p}, t)=\sum_{r, \bar{p}} \bar{f}_{r}(\bar{p}, t)=N(t) .
$$

Time differentiation of Eq. 26) leads to the following equation

$$
\frac{d f_{r}(\bar{p}, t)}{d t}=-\frac{e E(t) \varepsilon_{\perp}}{\omega^{2}(\bar{p}, t)} \operatorname{Re}\left\{\Phi_{r}(\bar{p}, t)\right\} .
$$

Herein, we have used the equation of motion (24) and evaluated the occurring commutator. The function $\Phi_{r}(\bar{p}, t)$ in Eq. (29) describes the creation and annihilation of an electron-positron pair in the external electric field $E(t)$ and is given as

$$
\Phi_{r}(\bar{p}, t)=<0_{i n}\left|D_{-\bar{p} r}(t) B_{\bar{p} r}(t)\right| 0_{i n}>.
$$


It is straightforward to evaluate the derivative of this function. Applying the equations of motion (24), we obtain

$$
\frac{d \Phi_{r}(\bar{p}, t)}{d t}=\frac{e E(t) \varepsilon_{\perp}}{2 \omega^{2}(\bar{p}, t)}\left[2 f_{r}(\bar{p}, t)-1\right]-2 i \omega(\bar{p}, t) \Phi_{r}(\bar{p}, t)
$$

where because of charge neutrality of the system, the relation $f_{r}(\bar{p}, t)=\bar{f}_{r}(\bar{p}, t)$ is used. The solution of Eq. (31) may be written in the following integral form

$$
\Phi_{r}(\bar{p}, t)=\frac{\varepsilon_{\perp}}{2} \int_{t_{0}}^{t} d t^{\prime} \frac{e E\left(t^{\prime}\right)}{\omega^{2}\left(\bar{p}, t^{\prime}\right)}\left[2 f_{r}\left(\bar{p}, t^{\prime}\right)-1\right] \mathrm{e}^{2 i\left[\Theta\left(\bar{p}, t^{\prime}\right)-\Theta(\bar{p}, t)\right]} .
$$

The functions $\Theta(\bar{p}, t)$ and $\Theta\left(\bar{p}, t^{\prime}\right)$ in Eq. (32) can be taken at $t_{0}$ (see the definition (15)). Hence with $A\left(t_{0}\right)=0$, the function $\Phi_{r}(\bar{p}, t)$ vanishes at $t_{0}$. Inserting Eq. (32) into the r.h.s of Eq. (29) we obtain

$$
\frac{d f_{r}(\bar{p}, t)}{d t}=\frac{e E(t) \varepsilon_{\perp}}{2 \omega^{2}(\bar{p}, t)} \int_{-\infty}^{t} d t^{\prime} \frac{e E\left(t^{\prime}\right) \varepsilon_{\perp}}{\omega^{2}\left(\bar{p}, t^{\prime}\right)}\left[1-2 f_{r}\left(\bar{p}, t^{\prime}\right)\right] \cos \left(2\left[\Theta(\bar{p}, t)-\Theta\left(\bar{p}, t^{\prime}\right)\right]\right) .
$$

Since the distribution function obviously does not depend on spin (33), we can define: $f_{r}=$ $f$. With the substitution $f(\bar{p}, t) \rightarrow F(\bar{P}, t)$, where the 3 -momentum is now defined as $\bar{P}\left(p_{\perp}, P_{\|}(t)\right)$, the kinetic equation (33) is reduced to its final form:

$$
\frac{d F(\bar{P}, t)}{d t}=\frac{\partial F(\bar{P}, t)}{\partial t}+e E(t) \frac{\partial F(\bar{P}, t)}{\partial P_{\|}(t)}=\mathcal{S}_{(-)}(\bar{P}, t)
$$

with the Schwinger source term

$$
\mathcal{S}_{(-)}(\bar{P}, t)=\frac{e E(t) \varepsilon_{\perp}}{2 \omega^{2}(\bar{p}, t)} \int_{-\infty}^{t} d t^{\prime} \frac{e E\left(t^{\prime}\right) \varepsilon_{\perp}}{\omega^{2}\left(\bar{p}, t^{\prime}\right)}\left[1-2 F\left(\bar{P}, t^{\prime}\right)\right] \cos \left(2\left[\Theta(\bar{p}, t)-\Theta\left(\bar{p}, t^{\prime}\right)\right]\right) .
$$

Recently in Ref 6, a kinetic equation similar to (34) has been derived within a projection operator formalism for the case of a time-independent electric field where it was first noted that this source term has non-Markovian character. As well as in this method the multiple pair creation is not considered. The presence of the Pauli blocking factor $[1-2 F(\bar{P}, t)]$ in the source term has been obtained earlier in Ref 18. We would like to emphasize the closed form of the kinetic equation in the present work where the source term does not include the anomalous distribution functions (30) for fermion-antifermion pair creation (annihilation).

\subsection{Creation of boson pairs}

In this subsection we derive the kinetic equation with source term for the case of pairs of charged bosons in a strong electric field.

The Klein-Gordon equation reads

$$
\left(\left(\partial^{\mu}+i e A^{\mu}\right)\left(\partial_{\mu}+i e A_{\mu}\right)+m^{2}\right) \phi(x)=0 .
$$

The solution of the Klein-Gordon equation in the presenef of the electric field defined by the vector potential $A_{\mu}=(0,0,0, A(t))$ is taken in the form 24

$$
\phi_{\bar{p}}^{( \pm)}(x)=[2 \omega(p)]^{-1 / 2} e^{i \bar{x} \bar{p}} g^{( \pm)}(\bar{p}, t),
$$


where the functions $g^{( \pm)}(\bar{p}, t)$ satisfy the oscillator-type equation with a variable frequency

$$
\ddot{g}^{( \pm)}(\bar{p}, t)+\omega^{2}(\bar{p}, t) g^{( \pm)}(\bar{p}, t)=0 .
$$

Solutions of Eq. (38) for positive and negative frequencies are defined by their asymptotic behavior at $t_{0}=t \rightarrow-\infty$ similarly to Eq. (7).

The field operator in the in-state is defined as

$$
\phi(x)=\int d^{3} p\left[\phi_{\bar{p}}^{(-)}(x) a_{\bar{p}}\left(t_{0}\right)+\phi_{\bar{p}}^{(+)}(x) b_{v p}^{+}\left(t_{0}\right)\right] .
$$

The diagonalization of the hamiltonian corresponding to the instantaneous stateis achieved by the transition to the quasiparticle representation. The Bogoliubov transformation for creation and annihilation operators of quasiparticles has the following form

$$
\begin{aligned}
& a_{\bar{p}}(t)=\alpha_{\bar{p}}(t) a_{\bar{p}}\left(t_{0}\right)+\beta_{\bar{p}}(t) b_{-\bar{p}}^{+}\left(t_{0}\right), \\
& b_{-\bar{p}}(t)=\alpha_{-\bar{p}}(t) b_{\bar{p}}\left(t_{0}\right)+\beta_{-\bar{p}}(t) a_{-\bar{p}}^{+}\left(t_{0}\right)
\end{aligned}
$$

with the condition

$$
\left|\alpha_{\bar{p}}(t)\right|^{2}-\left|\beta_{\bar{p}}(t)\right|^{2}=1
$$

The derivation of the Bogoliubov coefficients $\alpha$ and $\beta$ is similar to that given in the previous subsection. We obtain the equations of motion for the coefficients of the canonical transformation 40 as follows

$$
\begin{aligned}
\dot{\alpha}_{\bar{p}}(t) & =\frac{\dot{\omega}(\bar{p}, t)}{2 \omega(\bar{p}, t)} \beta_{\bar{p}}^{*}(t) e^{2 i \Theta(\bar{p}, t)}, \\
\dot{\beta}_{\bar{p}}(t) & =\frac{\dot{\omega}(\bar{p}, t)}{2 \omega(\bar{p}, t)} \alpha_{\bar{p}}^{*}(t) e^{2 i \Theta(\bar{p}, t)} .
\end{aligned}
$$

Following the derivation for the case of fermion production, we arrive at the final result for the source term in the bosonic case

$$
\mathcal{S}_{(+)}(\bar{P}, t)=\frac{e E(t) \varepsilon_{\perp}}{2 \omega^{2}(\bar{p}, t)} \int_{-\infty}^{t} d t^{\prime} \frac{e E\left(t^{\prime}\right) \varepsilon_{\perp}}{\omega^{2}\left(\bar{p}, t^{\prime}\right)}\left[1+2 F\left(\bar{P}, t^{\prime}\right)\right] \cos \left(2\left[\Theta(\bar{p}, t)-\Theta\left(\bar{p}, t^{\prime}\right)\right]\right),
$$

which differs from the fermion case just by the sign in front of the distribution function due to the different statistics of the produced particles.

\section{Discussion of the source term}

\subsection{Properties of the source term}

We can combine the results for fermions (35) and for bosons (44) into a single kinetic equation

$$
\frac{d F_{( \pm)}(\bar{P}, t)}{d t}=\frac{\partial F_{( \pm)}(\bar{P}, t)}{\partial t}+e E(t) \frac{\partial F_{( \pm)}(\bar{P}, t)}{\partial P_{3}}=\mathcal{S}_{( \pm)}(\bar{P}, t)
$$

Here, the upper (lower) sign corresponds to the Bose-Einstein (Fermi-Dirac) statistics. Based on microscopic dynamics, these kinetic equations are exact within the approximation of a time-dependent homogeneous electric field and the neglect of collisions. The Schwinger source terms (35) and (44) are characterized by the following features: 
1. The kinetic equations (45) are of non-Markovian type due to the explicit dependence of the source terms on the whole pre-history via the statistical factor $1 \pm 2 F(\bar{P}, t)$ for fermions or bosons, respectively. The memory effect is expected to lead to a modification of particle pair creation as compared to the (Markovian) low-density limit, where the statistical factor is absent.

2. The difference of the dynamical phases, $\Theta(\bar{p}, t)-\Theta\left(\bar{p}, t^{\prime}\right)$, under the integrals (35) and (44) generates high frequency oscillations.

3. The appearapce of such a source term leads to entropy production due to pair creation (see also Raul) and therefore the time reversal symmetry should be violated, but it does not result in any monotonic entropy increase (in absence of collisions).

4. The source term and the distribution functions have a non-trivial momentum dependence resulting in the fat that particles are produced not only at rest as assumed in previous studies, e.g. Ref 19 .

5. In the low density limit and in the simple case of a constant electric field we reproduce the pair production rate given by Schwinger's formula

$$
\mathcal{S}^{\mathrm{cl}}=\lim _{t \rightarrow+\infty}(2 \pi)^{-3} g \int d^{3} P \mathcal{S}(\bar{P}, t)=\frac{e^{2} E^{2}}{4 \pi^{3}} \exp \left(-\frac{\pi m^{2}}{|e E|}\right) .
$$

As noted above, Raul found that the source term has a non-Markovian behaviour by deriving the productipn rate within a projector method. In the limit of a constant field our results agree with Ref 6 . In our approach the electric field is treated as a general time dependent field and hence there is no a priori limitation to constant fields. However our result allows to explore the influence of any time-dependent electric field on the pair creation process. It is important to note that in general this time dependence should be given by a selfconsistent solution of the coupled field equations, namely the Dirac (Klein-Gordon) equation and the Maxwell equation. This would incorporate back reactions as mentioned in the introduction. However, the solution of such a system of equations is beyond the scope of this work. Herein we will restrict ourselves to the study of some features of the new source term.

Finally we remark that the source term is characterized by two time scales: the memory time

$$
\tau_{m e m} \sim \frac{\varepsilon_{\perp}}{e E}
$$

and the production interval

$$
\tau_{\text {prod }}=1 /<S_{( \pm)}>
$$

with $<S_{( \pm)}>$denoting the time averaged production rate. As long as $E<<m^{2} / e<\varepsilon_{\perp}^{2} / e$, the particle creation precess is Markovian: $\tau_{m e m}<<\tau_{\text {prod }}$. This results for constant fields agree with those of Raul 6 .

\subsection{Numerical results}

In order to study the new source term, we consider two different cases, namely a constant field and a time dependent electric field. For the numerical evaluation we start with Eq. (35) assuming a dilute system, $F=0$, and

$$
\tilde{\mathcal{S}}\left(\tilde{p}_{\|}, \tilde{t}\right)=\frac{S\left(\tilde{p}_{\|}, \tilde{t}\right)}{\varepsilon_{\perp}}=\frac{\tilde{E}(\tilde{t})}{2 \tilde{\omega}^{2}\left(\tilde{p}_{\|}, \tilde{t}\right)} \int_{-\infty}^{\tilde{t}} d \tilde{t}^{\prime} \frac{\tilde{E}\left(\tilde{t}^{\prime}\right)}{\tilde{\omega}^{2}\left(\tilde{p}_{\|}, \tilde{t}^{\prime}\right)} \cos \left(2\left[\Theta\left(\tilde{p}_{\|}, \tilde{t}\right)-\Theta\left(\tilde{p}_{\|}, \tilde{t}^{\prime}\right)\right]\right),
$$




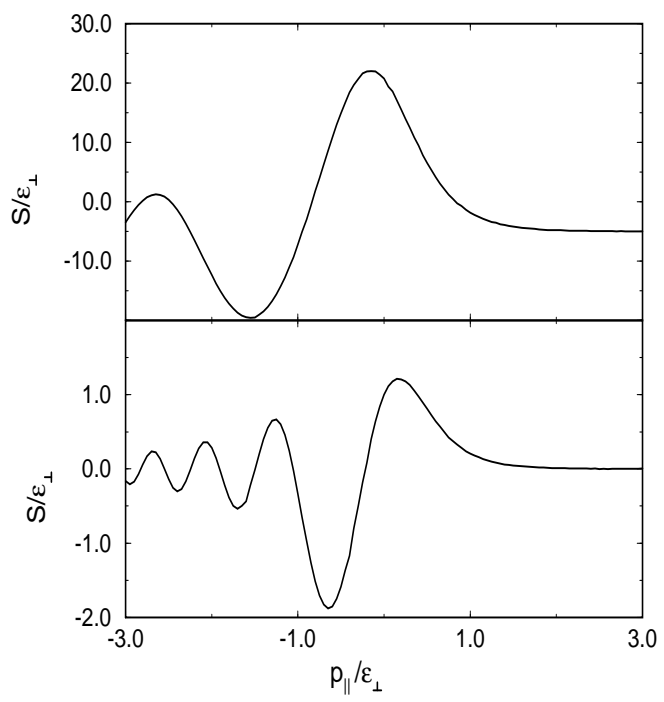

Fig. 1. The pair production rate as a function of parallel momentum for a constant, strong field (upper plot: $\tilde{E}_{0}=1.5$ ) and a weak field (lower plot: $\tilde{E}_{0}=0.5$ ) at $\tilde{t}=0$.

where we have introduced dimensionless variables

$$
\begin{gathered}
\tilde{E}(\tilde{t})=e E(\tilde{t}) / \varepsilon_{\perp}^{2}, \quad \quad \tilde{t}=t \varepsilon_{\perp}, \\
\tilde{p}_{\|}=p_{\|} / \varepsilon_{\perp}, \quad \tilde{\omega}=\omega / \varepsilon_{\perp} .
\end{gathered}
$$

This notation is convenient to distinguish the weak field $(\tilde{E}<1)$ and strong field $(\tilde{E}>1)$ limits. A particularly simple result is obtained if we assume a constant field,

$$
\tilde{A}(\tilde{t})=A(\tilde{t}) / \varepsilon_{\perp}=\tilde{t} \tilde{E}_{0} / e
$$

where the electric field does not depend on time and the energy is given as

$$
\tilde{\omega}_{0}^{2}\left(\tilde{p}_{\|}, \tilde{t}\right)=1+\left(\tilde{p}_{\|}-\tilde{E}_{0} \tilde{t}\right)^{2} .
$$

For the source term we obtain

$$
\tilde{\mathcal{S}}\left(\tilde{p}_{\|}, \tilde{t}\right)=\frac{\tilde{E}_{0}^{2}}{2 \tilde{\omega}_{0}^{2}\left(\tilde{p}_{\|}, \tilde{t}\right)} \int_{-\infty}^{\tilde{t}} d \tilde{t}^{\prime} \frac{1}{\tilde{\omega}_{0}^{2}\left(\tilde{p}_{\|}, \tilde{t}^{\prime}\right)} \cos \left(2 \int_{\tilde{t}^{\prime}}^{\tilde{t}} d \tilde{t}^{\prime \prime} \tilde{\omega}_{0}\left(\tilde{p}_{\|}, \tilde{t}^{\prime \prime}\right)\right) .
$$

In Fig. 11 we plot the particle production rate as function of the parallel momentum $p_{\|}$for a weak constant field and a strong field, respectively. The rates are normalized to be of the order of one for $\tilde{E}_{0}=0.5$ at zero values of both momentum and time. The production rate is positive when particles are produced with positive momenta. Pairs with negative momenta are moving against the field and hence get annihilated, clearly to be seen in the negative production rate. These results mainly agree with those of Ref. 


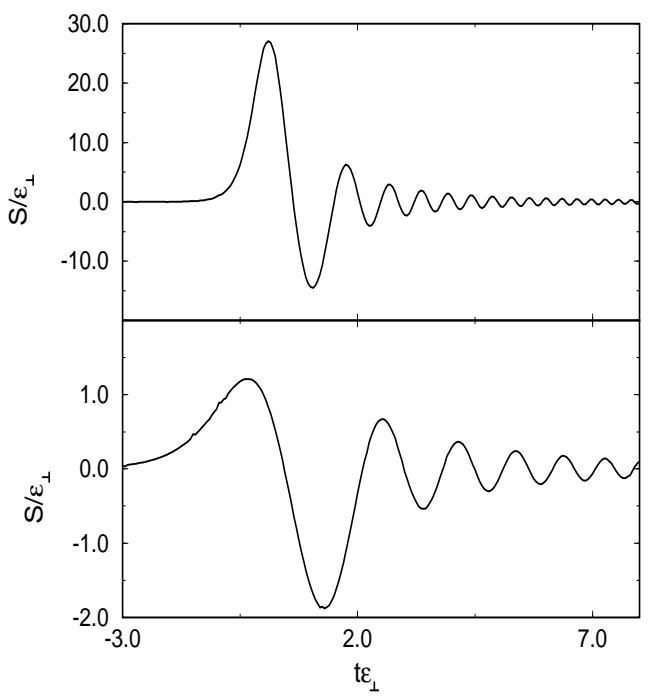

Fig. 2. The pair production rate as a function of time for a constant, strong field (upper plot: $\tilde{E}_{0}=1.5$ ) and a weak field (lower plot: $\tilde{E}_{0}=0.5$ ) at zero parallel momentum.

considered. Note that using thf prefactor and field strengths chosen by Rau, we reproduce exactly the results given in Ref.6.

fn considering the time dependence of the source term, we go beyond the analysis of Ref6. In Fig. 2, we display the time dependence of the production rate at zero momentum. The maximum of the production rate is concentrated around zero and shows an oscillating behaviour for large times. Indeed it is possible to write Eq. (54) in terms of the Airy function because the constant field provides an analytical solution for the dynamical phase difference using the energy given in Eq. (53). The production of pairs for strong fields is larger than that of weak fields, and the typical time period of the oscillations is smaller.

The situation changes if we allow the electric field to be time dependent. We assume a Gaussian field at the dimensionsless time $\tau$ with the width $\tilde{\sigma}=\sigma \varepsilon_{\perp}$,

$$
\tilde{E}(\tilde{t})=\tilde{E}_{0} e^{-(\tilde{t}-\tau)^{2} / \tilde{\sigma}^{2}}
$$

and obtain

$$
\tilde{A}(\tilde{t})=-\tilde{E}_{0} \sigma \frac{\sqrt{\pi}}{2}\left[\operatorname{Erf}[(\tilde{t}-\tau) / \tilde{\sigma}]-\operatorname{Erf}\left[\left(-\tau-t_{0}\right) / \tilde{\sigma}\right]\right] .
$$

The occuring error function is defined as

$$
\operatorname{Erf}(z)=\frac{2}{\sqrt{\pi}} \int_{0}^{z} e^{-x^{2}} d x
$$

Using this Ansatz for the field strength in Eq. (49) we obtain the numerical results plotted in Figs. 3 and 4 . Therein all occuring values are dimensionsless. The electric field is non 


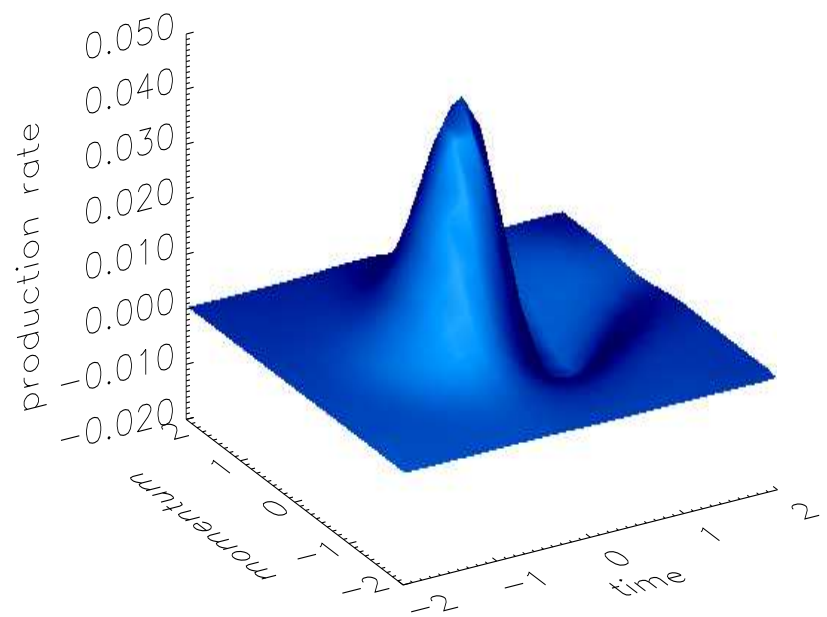

Fig. 3. The pair production rate, $\tilde{S}\left(\tilde{p}_{\|}, \tilde{t}\right)$, as a function of time and parallel momentum for a time dependent weak electric field charactarized by $\tilde{E}_{0}=0.5, \tilde{\sigma}=1$ and $\tau=0$. All plotted values are dimensionless as described in the text.

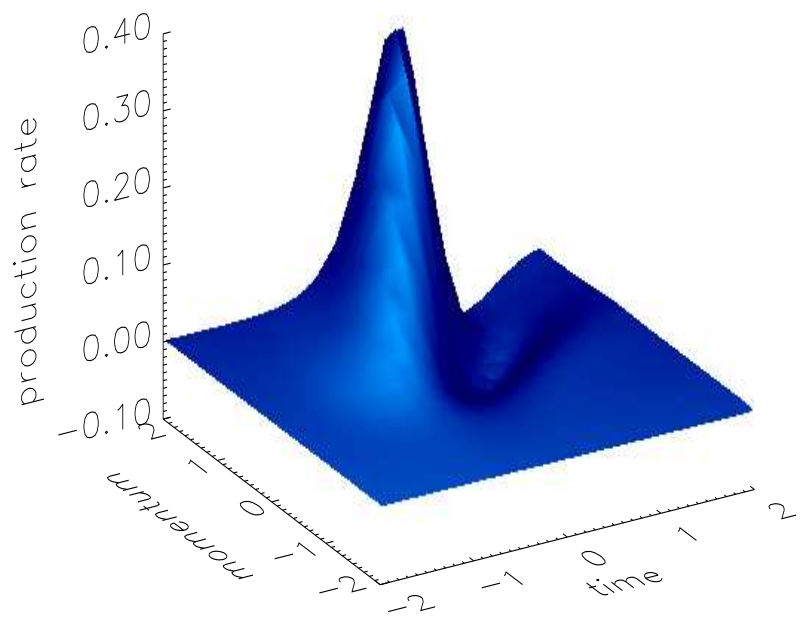

Fig. 4. The pair production rate, $\tilde{S}\left(\tilde{p}_{\|}, \tilde{t}\right)$, as a function of time and parallel momentum for a time dependent strong electric field charactarized by $\tilde{E}_{0}=1.5, \tilde{\sigma}=1$ and $\tau=0$. All plotted values are dimensionless as described in the text. 
zero within a certain width $\tilde{\sigma}=1$ for $\tau=0$ around $\tilde{t}=0$. Therefore the oscillations for times beyond the time where the electrical field is finite are damped out. The pair production rate is peaked around small momenta for both weak and strong fields. For strong fields the distribution is shifted to positive momenta but remains still close to small parallel momenta. It is important to point out that the production of particles happens not only at rest $\left(p_{\|}=0\right)$ what is assumed in many works also addressing the back reaction problem, e.g.19. In contrary we find a non-trivial momentum dependence of the pair creation rate depending on the field strength and on time.

\section{Summary}

We have derived a quantum kinetic equation within a consistent field theoretical treatment which contains the creation of particle-antiparticle pairs in a time-dependent homogeneous electric field. For both fermions and bosons we obtain a source term providing a kinetic equation of non-Markovian character. The source term is characterized by a pair production rate that contains a time integration over the evolution of the distribution function and therefore involves memory effects.

For the simple case of a constant electric field in low density limit and Markgrian approximation, we analytically and numerically reproduce the results of earlier works 6 . Since our approach is not restricted to constant fields, we have explored the dependence of the source term on a time dependent (model) electric field with a Gaussian shape. Within these two different Ansätze for the field, we have performed investigations of the time structure of the source term. The production of pairs does not happen at rest only. We observe a non-trivial momentum dependence of the source term depending on the field strength and on time.

The particle production source is dominated by two time scales: the memory time and the production time. The numerical results mainly show oscillations due to the dynamical phase and urge the need to include the Maxwell equation to determine the electric field by physical boundary conditions (back reactions), work in this direction is in progress. Furthermore, it would be of great interest to extend this approach to the QCD case to explore the impact of a non-Markovian source term on the pre-equilibrium physics in ultrarelativistic heavy-ion collisions.

\section{Acknowledgment}

The authors wish to thank J.M. Eisenberg, V.G. Morozov and J. Rau for valuable discussions and comments. The authors gratefully acknowledge the hospitality at the Tel Aviv University (S.S.), the University of Rostock (S.A.S.), the JINR Dubna (D.B. and G.R.) and the GSI Darmstadt (V.D.T.) where part of this work has been carried out. This work was supported in part by the Russian State Committee of High-School Education under grant No. 95-0-6/1-53, the Heisenberg-Landau program, the WTZ program of the BMBF and by the HSP-III under the project No. 00376003.

\section{References}

1. N.B. Naroshni and A.I. Nikishov, Yad. Fiz. 11 (1970) 1072 (Sov. J. Nucl. Phys. 11 (1970) 596); V.S. Popov and M.S. Marinov, Yad. Fiz. 16 (1972) 809 (Sov. J. Nucl. Phys. 16 (1974) 449); V.S. Popov, Zh. Eksp. Teor. Fiz. 62 (1972) 1248 (Sov. Phys. JETP 35 (1972) 659); M.S. Marinov and V.S. Popov, Fort. d. Phys. 25 (1977) 373. 
2. A. Białas and W. Czyż, Phys. Rev. D 30 (1984) 2371; 31 (1985) 198; Z. Phys. C 28 (1985) 255; Nucl. Phys. B 267 (1985) 242; Acta Phys. Pol. B17 (1986) 635.

3. K. Kajantie and T. Matsui, Phys. Lett. B 164 (1985) 373.

4. G. Gatoff, A.K. Kerman, and T. Matsui, Phys. Rev. D 36 (1987) 114.

5. F. Cooper, J.M. Eisenberg, Y. Kluger, E. Mottola, and B. Svetitsky, Phys. Rev. D 48 (1993) 190.

6. J. Rau, Phys.Rev. D 50 (1994) 6911.

7. R.S. Bhalerao and V. Ravishankar, Phys.Lett. B 409 (1997) 38.

8. W. Greiner, B. Müller, and J. Rafelski, Quantum Electrodynamics of Strong Fields (Springer-Verlag, Berlin, 1985).

9. A.A. Grib, S.G. Mamaev, and V.M. Mostepanenko, Vacuum quantum effects in strong external fields, (Atomizdat, Moscow, 1988).

10. S. Nussinov, Phys. Rev. Lett. 34 (1975) 1286.

11. B. Andersson, G. Gustafson, G. Ingelman, and T. Sjostrand, Phys. Rep. 97 (1993) 31.

12. N.K. Glendenning and T. Matsui, Phys. Rev. D 28 (1983) 2890.

13. F. Sauter, Z. Phys. 69 (1931) 742.

14. W. Heisenberg and H. Euler, Z. Phys. 98 (1936) 714.

15. J. Schwinger, Phys. Rev. 82 (1951) 664.

16. F. Cooper and E. Mottola, Phys. Rev. D 40 (1989) 456.

17. Y. Kluger, J.M. Eisenberg, B. Svetitsky, F. Cooper, and E. Mottola, Phys. Rev. Lett. 67 (1991) 2427.

18. Y. Kluger, J.M. Eisenberg, B. Svetitsky, F. Cooper, and E. Mottola, Phys. Rev. D 45 (1992) 4659.

19. Y. Kluger, J.M. Eisenberg, and B. Svetitsky, Int. J. Mod. Phys. E 2 (1993) 333 (here further references may be found).

20. F. Cooper, S. Habib, Y. Kluger, E. Mottola, J.P. Paz, and P.R. Anderson, Phys. Rev. D 50 (1994) 2848.

21. J.M. Eisenberg, Phys. Rev. D 51 (1995) 1938.

22. Ch. Best and J.M. Eisenberg, Phys. Rev. D 47 (1993) 4639.

23. J. Rau and B. Müller, Phys. Rep. 272 (1996) 1.

24. J.D. Bjorken and S.D. Drell, Relativistic Quantum Mechanics (McGraw-Hill, New York, 1964). 